\title{
A Particle Method for the $b$-Equation
}

\author{
Zhiqiang Xing*, Yong Duan*, Huiyuan Wu \\ School of Mathematical Sciences, University of Electronic Science and Technology of China, Chengdu, China \\ Email: ${ }^{*}$ 18030465450@163.com, ${ }^{*}$ duanyong72@aliyun.com
}

Received June 2014

\begin{abstract}
In this paper, we apply the particle method to solve the numerical solution of a family of non-linear Evolutionary Partial Differential Equations. It is called $b$-equation because of its bi-Hamiltonian structure. We introduce the particle method as an approximation of these equations in Lagrangian representation for simulating collisions between wave fronts. Several numerical examples will be set to illustrate the feasibility of the particle method.
\end{abstract}

\section{Keywords}

Particle Method, $b$-Equation, Peakon Solution, Lagrangian Representation

\section{Introduction}

In the past decades, the particle method has become one of the most important techniques to solve the particle differential equation (PDEs). Most researches have focused on the development of the application on the mathematics and physical fields [1] [2]. In these methods, a solution of a given equation is represented by a collection of particles, which are located in points $x_{i}$ and carry masses $p_{i}$. Equation of evolution in time is written to describe the dynamics of the location of the particles and these weights. Because of the Lagrangian dynamic for the method, small scale that might develop in a solution can be easily described with a relatively small number of particles. In our work, we present the particle method for approximating solution of our equations. Now we investigate the Cauchy problems of the $b$-equation in one-dimensional cases. For $x \in R, t>0$

$$
\partial_{t} m+\partial_{t}(u m)+(b-1) m \partial_{x} u=0
$$

where $b>0$ and the momentum $m$ and velocity $u$ are functions of the time variable $t$ and the variables $x$. They are related by the second-order Helmholtz operator,

with length scale $\alpha$

$$
m=u-\alpha^{2} \Delta u
$$

The velocity function $u(x, t)$ can also be expressed as a convolution of $m(x, t)$ with the kernel $G(x)$,

$$
u(x, t)=G * m=\int_{R} G(x-y) m(y, t) \mathrm{d} y
$$

It is well-known that the Equation (2) has solitary wave solutions of the form $u(x, t)=a G(x-c t)$ with

\footnotetext{
*orresponding authors.
} 
speed $c=-a G(0)$, which is proportional to the amplitude of the solution. To our knowledge, a solution is represented by a collection of particles, which are located in points $x_{i}$ and carry masses $p_{i}$. So, denoting the time derivative of $x(\xi, t), p(\xi, t)$, by $x^{\prime}(\xi, t), p^{\prime}(\xi, t)$ and regarding $\xi$ (we call it Lagrangian label) as a parameter, the Lagrangian dynamics of the $b$-equation is given by,

$$
\begin{aligned}
& x^{\prime}(\xi, t)=\int_{-L}^{L} G(x(\xi, t)-x(\eta, t)) p(\eta, t) \mathrm{d} \eta, \\
& p^{\prime}(\xi, t)=-(b-1) p(\xi, t) \int_{-L}^{L} G^{\prime}(x(\xi, t)-x(\eta, t)) p(\eta, t) \mathrm{d} \eta .
\end{aligned}
$$

with initial data $x(\xi, 0)=\xi p(\xi, 0)=m_{0}(x(\xi, t))=m_{0}(\xi)$. The solution is then found by following the time evolution of the locations and the weights of these particles according to a system of ODEs obtained by considering a weak formulation of the problem. In this paper, we take

$$
G(x)=\frac{1}{2 \alpha} \mathrm{e}^{-x / \alpha}
$$

where, $\alpha$ is the length scale of kernel and $G(x)$ is the fundamental solution for Helmholtz operator $1-\alpha^{2} \partial_{x x}$, i.e. $\left(1-\alpha^{2} \partial_{x x}\right) G=\delta$ with $\delta$ representing the Dirac $\delta$ distribution.

The purpose of this paper is to present the particle method in Lagrangian representation for simulating collisions and to study of the dynamic of $N$ points governed by the equation. Several numerical examples will be set to illustrate the feasibility of the particle method.

Besides above, we present a brief overview of the particle method and some of its main properties which are necessary for the study of numerical collisions among peakon solutions in Section 2. Then, we apply lots of numerical examples to verify our particle method in Section 3. According to the numerical results, a conclusion is given in the last section.

\section{Description of the Particle Method for the $b$-Equation}

In this section, we consider the $b$-equation (1). As mentioned above, Equation (1) coincides with the dispersionless C-H equation in [3] and D-P equation in [4]. It was first derived by using asymptotic expansions directly in the Hamiltonian for Euler's equations in the shallow water regime. The equation is completely integrable and possesses solitary solutions [5]. We briefly describe a particle method for the equation. For the specific description on the particle method for Equation (1), we refer the reader to [1] [2] [6]. We begin with searching for a weak solution in the form of a linear collection of Dirac delta distributions. In particular, we look for a solution of the form,

$$
m^{N}(x, t)=\sum_{i=1}^{N} p_{i}(t) \delta\left(x-x_{i}(t)\right) .
$$

Here, $x_{i}(t)$ and $p_{i}(t)$ represent the location of the $i$-th particle and its weight, and $N$ denote the total number of particles. The solution is then found by following the time evolution of the locations and the weights of the particles according to the following system of ODEs,

$$
\begin{aligned}
& \frac{\mathrm{d} x_{i}(t)}{\mathrm{d} t}=u\left(x_{i}(t), t\right), \\
& \frac{\mathrm{d} p_{i}(t)}{\mathrm{d} t}+u_{x}\left(x_{i}(t), t\right) p_{i}(t)=0 .
\end{aligned}
$$

Here, $i=1,2, \cdots N$ The velocity $u$ in Equation (5) is obtained by convolution $u=G * m$ with the Green's function,

$$
G(|x-y|)=\frac{1}{2 \alpha} \mathrm{e}^{-|x-y| / \alpha},
$$

for the 1-D Helmholtz operator that relates $m$ and $u$. Consequently, the velocity and its derivative corresponding to the solution (5) are given by 


$$
u(x, t)=\frac{1}{2} \sum_{i=1}^{N} p_{i}(t) \mathrm{e}^{-\left|x-x_{i}(t)\right| / \alpha}
$$

and

$$
u_{x}(x, t)=-\frac{1}{2} \sum_{i=1}^{N} p_{i}(t) \operatorname{sgn}\left(x-x_{i}(t)\right) \mathrm{e}^{-\left|x-x_{i}(t)\right| / \alpha} .
$$

In order to initiate the time integration, we should choose the initial positions and weights $\left(x_{i}(0), p_{i}(0)\right)$ in such a way that for any test $\phi(x) \in C_{0}^{\infty}(R)$, we have that

$$
\left\langle m_{0}^{N}(\cdot), \phi(\cdot)\right\rangle=\int_{R} m_{0}(x) \phi(x) \mathrm{d} x \approx \sum_{i=1}^{N} p_{i}(0) \phi\left(x_{i}\right),
$$

where

$$
m_{0}^{N}(x)=\sum_{i=1}^{N} p_{i}(0) \delta\left(x-x_{i}(0)\right)
$$

One way of solving the last equation can be, e.g. to cover the computational domain $R$ with a uniform mesh of spacing $\Delta x>0$ and denote by $C_{i}$ the interval

$$
C_{i}=\left[x_{i-1 / 2}, x_{i+1 / 2}\right]=\left\{x \mid x_{i-1 / 2} \leq x \leq x_{i+1 / 2}\right\},
$$

Here, $i=1,2, \cdots N$ and $x_{i}(0)=i \Delta x$ in the center $C_{i}$. For example, a midpoint quadrature is then given by setting $p_{i}(0)=\Delta x m_{0}\left(x_{i}\right)$. In practice, except for very special cases, the functions $x_{i}(t)$ and $p_{i}(t)$ have to be determined numerically and the system (8) - (9) must be integrated by an appropriate ODE solver. For our numerical experiments, we consider a strong-stability preserving Runge-Kutta method to solve the ODEs.

Obviously we can obtain the solution $\left(u_{h}(x, t), m_{h}(x, t)\right)$, the numerical approximation of $(u(x, t), m(x, t))$. Furthermore, if the initial data assumes the form of a peakon, then the peakon solution generated is exact. Then any errors emanate solely from the ODE solver.

\section{Properties of the Particle System}

Now, we present some general properties of the particle system which are pertinent to our paper to research on the investigation of collisions of peakons.

- One can verify that the function $x_{i}(t)$ and $p_{i}(t)$ in (6) satisfy that canonical Hamiltonian equations

$$
\frac{\mathrm{d} x_{i}}{\mathrm{~d} t}=\frac{\partial H^{N}}{\partial p_{i}}, \quad \frac{\mathrm{d} p_{i}}{\mathrm{~d} t}=\frac{\partial H^{N}}{\partial x_{i}}, \quad i=1,2, \cdots N .
$$

where $H^{N}$ is the Hamiltonian function defined as

$$
H^{N}(t ; x, p)=\frac{1}{4 \alpha} \sum_{i=1}^{N} \sum_{j=1}^{N} p_{i}(t) p_{j}(t) \mathrm{e}^{-\left|x_{i}(t)-x_{j}(t)\right| / \alpha}
$$

- Another important conservation law is that the total momentum of the particle system is conserved as follows

$$
\frac{\mathrm{d}}{\mathrm{d} t}\left[\sum_{i=1}^{N} p_{i}(t)\right]=0
$$

- Finally, if the initial moment given in Equation (5) are positive, i.e. $p_{i}(0)>0$ for all $i=1,2, \cdots N$, then $p_{i}(t)>0$ for all $t>0$. In addition, if $x_{i}(0)<x_{i+1}(0) i=1,2, \cdots N$, then the particles never cross, i.e. $x_{i}(0)<x_{i+1}(0)$ for any $i=1,2, \cdots N$, and for all $t$. This important property was proved in [7] by using a Lax-Pair formulation. It can also be proved by using a conservation law, 


$$
\frac{\mathrm{d} P_{N}(t)}{\mathrm{d} t}=\frac{\mathrm{d}}{\mathrm{d} t}\left(\prod_{k=1}^{N} p_{k}(t) \prod_{k=1}^{N-1}\left[G(0)-G\left(x_{k}(t)-x_{k+1}(t)\right)\right]\right)=0
$$

as it has been in [6].

\section{Numerical Example}

In this section, we present several numerical simulations of application of the particle method to the numerical solution of Equation (1). These examples below test the method on a travelling wave solution for the dispersive case $b \neq 1$. We aim to illustrate the efficiency and the particle method.

\section{Smooth Travelling Wave Solution $b \neq 1$}

An explicit exact solution of Equation (1) is $u(x, t)=U(x-c t) \equiv U(s)$ [8], where $c=8 k / 3, k=b-1$ and $U(s)$ is given by

$$
u(s)=c\left(1-\frac{3 \sqrt{3}+6 \sin 2 z}{(1+2 \cos 2 z)(2 \sqrt{3} \cos 2 z-\sqrt{3} \cos 4 z+2 \sin 2 z+\sin 4 z)}\right)
$$

with $z=\arctan \left(\mathrm{e}^{s / 2}\right) / 3$ The initial condition $u_{0}(x)=U(x)$ yields the initial data for the particle algorithm $x_{i}^{0}=i \Delta x, p_{i}^{0}=k+m_{0}\left(x_{i}\right)$, where

$$
m_{0}(x)=k\left(\frac{c^{2}}{(c-U(x))^{2}}-1\right)
$$

With the initial condition, we compare the numerical solution computed by the particle method with the exact travelling wave solution to illustrate the order of convergence of the method. We remark that a fixed ratio $\Delta t / h=1 / b$ (step-size $h>0$ ) is used for all calculations throughout this paper. This fixed ratio is for the purpose of numerical convergence tests. We chose the temporal discretization to be compliant with the choice of $h$ used in the Riemann sum for the ODE system (6). Based on our experiments, we found that $\Delta t / h=1 / b$ would suffice for all simulations in this paper to prevent the onset of numerical instability. For the travelling wave simulation we take the constant $b=2,3$ The time integration proceeds through an explicit fourth-order R-K method. As indicated in Equation (8), using the $x_{i}$ and $p_{i}$ obtained from the ODE system, the numerical solution for the PDE is then set on the physical domain. We study the numerical error between exact and computed solutions with $l_{2}$-norm

$$
\|\varepsilon\|=\sqrt{\Delta x \sum_{i=1}^{N}\left|u(j, t)-u_{h}^{\varepsilon}(j, t)\right|^{2}}=\sqrt{\Delta x \sum_{i=1}^{N} \varepsilon^{2}}
$$

where $\Delta x$ is the grid size for the mesh on the physical domain, defined as $\Delta x=L / N$, with $L$ the length of the physical domain and $N$ is the number of grid points. This $\Delta x$ is independent of the increment $h$ used in the Riemann sum for the ODE system, which is defined as $h=L / N$ In the mesh-refinement study, for a fixed domain, we vary the size of $h$, hence conventionally we choose $\Delta x$ to be the same size as $h$ so that for simplicity the number of grid points is the same as the number of particles.

Example 1: Figure 1 is a plot for the computed travelling wave solution at time $t=20$ on a domain $[-100,100]$, confirming that the particle method is second-order accurate in space and there is no discrepancy between the numerical solutions or the particle method. On a fixed domain $[-30,30]$, at time $t=1$, the errors are listed in Table 1.

Example 2: Figure 2 is another plot for the computed travelling wave solution at time $t=10$ on a domain $[-100,100]$, confirming that the particle method is second-order accurate in space and there is no discrepancy between the numerical solutions and the particle method. On a fixed domain $[-30,30]$, at time $t=1$, the errors are listed in Table 2. As the two tables show, the particle method is second-order accurate in space, consistently with our theory. 


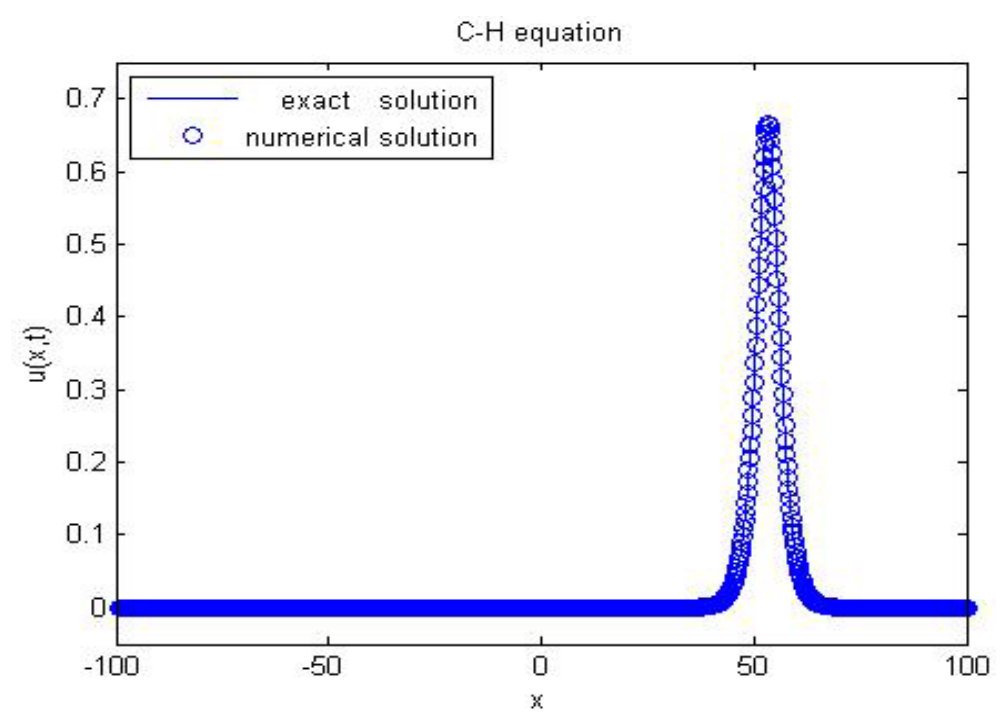

Figure 1. Numerical simulations of the travelling wave solution (15) with $k=1$ when $N=1000 h=0.1, t=20$.

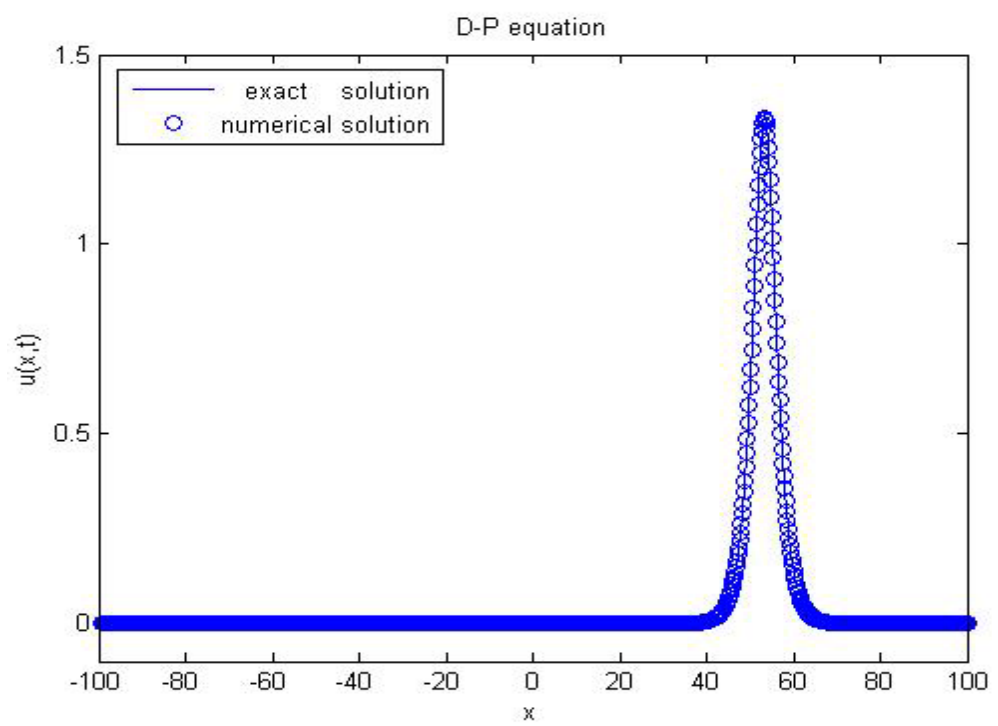

Figure 2. Numerical simulations of the travelling wave solution (15) with $k=2$ when $N=1000 h=0.1, t=10$.

Table 1. When $t=1 ; k=1$, Convergence rate for the particle method.

\begin{tabular}{cccccc}
\hline$\Delta x$ & 0.1 & 0.05 & 0.025 & 0.0125 & 0.00625 \\
$\left\|u-u_{\text {exact }}\right\|$ & $6.80 \mathrm{e}-03$ & $1.70 \mathrm{e}-03$ & $4.22 \mathrm{e}-04$ & $1.06 \mathrm{e}-04$ & $2.62 \mathrm{e}-05$ \\
Rate & & 2.00 & 2.01 & 1.96 & 2.01 \\
\hline
\end{tabular}

Table 2. When $t=1 ; k=2$, Convergence rate for the particle method.

\begin{tabular}{cccccc}
\hline$\Delta x$ & 0.1 & 0.05 & 0.025 & 0.0125 & 0.00625 \\
$\left\|u-u_{\text {exac }}\right\|$ & 0.0306 & $7.60 \mathrm{e}-03$ & $1.90 \mathrm{e}-03$ & $4.76 \mathrm{e}-04$ & $1.19 \mathrm{e}-04$ \\
Rate & & 2.01 & 2.00 & 2.01 & 2.00 \\
\hline
\end{tabular}




\section{Conclusion}

In this paper, we established the mathematical theory of the Lagrangian dynamics for the $b$-equation with a special choice of the convolution kernel $G$ and under a suitable class of initial data. To a numerical implementation of the method, it can be approximated by uniformly distributing particles. In the end, we obtained the numerical solution though examples and specific data.

\section{Acknowledgements}

This work was supported by NSFC11171054.

\section{References}

[1] Camassa, R., Huang, J. and Lee, L. (2006) Integral and Integrable Algorithms for a Nonlinear Shallow-Water Wave Equation. Journal of Computational Physics, 216, 547-572. http://dx.doi.org/10.1016/j.jcp.2005.12.013

[2] Chertock, A., Du Toit, P. and Marsden, J. (2012) Integration of the EPDiff Equation by Particle Methods. ESAIM: Mathematical Modelling and Numerical Analysis, 46, 515-534. http://dx.doi.org/10.1051/m2an/2011054

[3] Chertock, A., Liu, J.G. and Pendleton, T. (2014) Elastic Collisions among Peakon Solutions for the Camassa-Holm Equation. Applied Numerical Mathematics, in press. http://dx.doi.org/10.1016/j.apnum.2014.01.001

[4] Matsuo, T. and Miyatake, Y. (2012) Conservative Finite Difference Schemes for Degasperis-Procesi Equation. Journal of Computational and Applied Mathematics, 236, 3728-3740. http://dx.doi.org/10.1016/j.cam.2011.09.004

[5] Camassa, R. and Lee, L. (2007) A Completely Integrable Particle Method for a Nonlinear Shallow-Water Wave Equation in Periodic Domains. Syst. Ser. A Math. Anal, 14, 1-5.

[6] Chertock, A., Liu, J.G. and Pendleton, T. (2012) Convergence of a Particle Method and Global Weak Solutions of a Family of Evolutionary PDEs. SIAM Journal on Numerical Analysis, 50, 1-21. http://dx.doi.org/10.1137/110831386

[7] Camassa, R., Huang, J. and Lee, L. (2005) On a Completely Integrable Numerical Scheme for a Nonlinear ShallowWater Wave Equation. Journal of Nonlinear Mathematical Physics, 12, 146-162. http://dx.doi.org/10.2991/jnmp.2005.12.s1.13

[8] Holden, H. and Raynaud, X. (2006) Convergence of a Finite Difference Scheme for the Camassa-Holm Equation. SIAM Journal on Numerical Analysis, 44, 1655-1680. http://dx.doi.org/10.1137/040611975 